\title{
Pattern of Intracranial Tuberculomas in Kuwait: A Computed Tomographic Study
}

\author{
Salwa Ramadan Aduh Prosper Sayeed Al-Muhtaseb Sukhpal Sawhney \\ Petkovska Liljana
}

Department of Radiology, Al-Adan Hospital, Kuwait

\section{Key Words}

Intracranial tuberculoma - Computed tomography ·

Antituberculous therapy · Kuwait

\begin{abstract}
Objective: To study the spectrum of computed tomographic (CT) morphologies of intracranial tuberculomas and to show the role of CT in evaluating the response to medical management. Subjects and Methods: The case files and CT scans of 20 patients with proven intracranial tuberculomas over a period of 15 years (1985-1999) were reviewed retrospectively. All the patients had pre- and post-contrast axial CT scans of the brain. Follow-up CT studies were done 2-12 months after starting antituberculous chemotherapy (ATT). Results: There were 15 males and 5 females. Single lesions were seen in $65 \%$ of patients. The majority of the lesions (78\%) were in the frontal and parietal lobes. Rare sites of presentation included midbrain and basal ganglia. The lesions were isodense in $81 \%$ of the precontrast studies and all were enhanced after intravenous administration of the contrast medium. Severe mass effect, hydrocephalus and meningeal enhancement were not common findings. In $80 \%$ of the patients the follow-up CT scans showed evi-
\end{abstract}

\section{KARGER}

Fax + 41613061234

E-Mail karger@karger.ch

www. karger.com

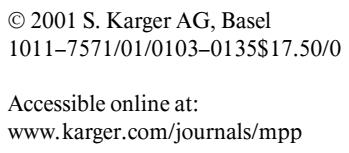

dence of response to ATT. Conclusion: CT is a reliable modality in the diagnostic workup and follow-up of patients with intracranial tuberculomas. Administration of contrast medium is mandatory for the evaluation of such patients.

Copyright $@ 2001$ S. Karger AG, Basel

\section{Introduction}

Neurotuberculosis remains a major health problem, both in the underdeveloped and developed countries [1, 2]. The reported incidence of intracranial tuberculosis (ICTb) in Kuwait is $1.4 \%$ of all intracranial space-occupying lesions [3]. Neurotuberculosis is believed to be secondary to haematogenous spread from extracranial tuberculous lesions [4]. When a patient presents with the clinical picture of an intracranial pathology and is from an area where tuberculosis ( $\mathrm{Tb})$ is endemic or has any evidence of extracranial $\mathrm{Tb}$ a diagnosis of ICTb should be suspected. The presence of such extracranial lesions helps as a diagnostic pointer. However its absence does not rule out ICTb [5]. Since Kuwait is a cosmopolitan state with a lot of migrant labour from developing countries where $\mathrm{Tb}$ is endemic, we conducted a study to establish the inci-
Dr. Salwa Ramadan M. Redha, MB BCh, FFR-RCSI

Department of Radiology, Al-Adan Hospital

PO Box 46969

Fahaheel 64020 (Kuwait)

Tel. +965 9761090, Fax +965 3941638, E-Mail salwafais@yahoo.com 
1

Fig. 1. CECT showing multiple small discenhancing lesions in both frontal and parietal lobes. Note the lesion in the right basal ganglia (arrow).

Fig. 2. CECT showing a small disc-enhancing lesion in the right side of the upper midbrain with no oedema or mass effect. It was not visualized on NECT scan.

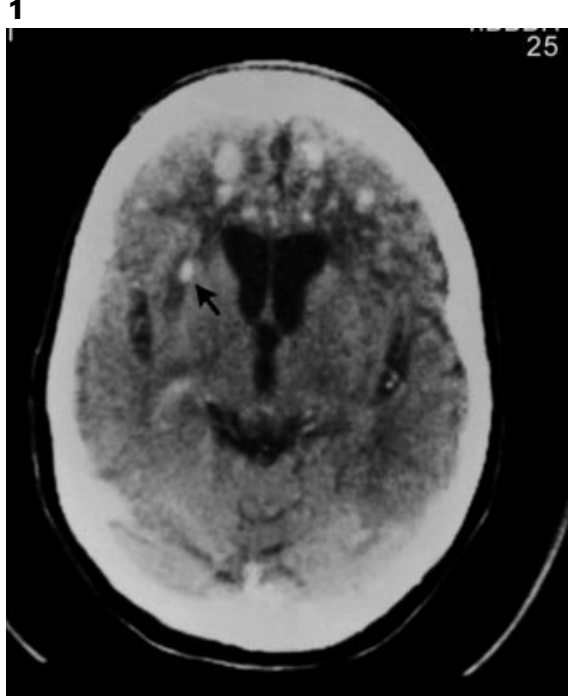

2

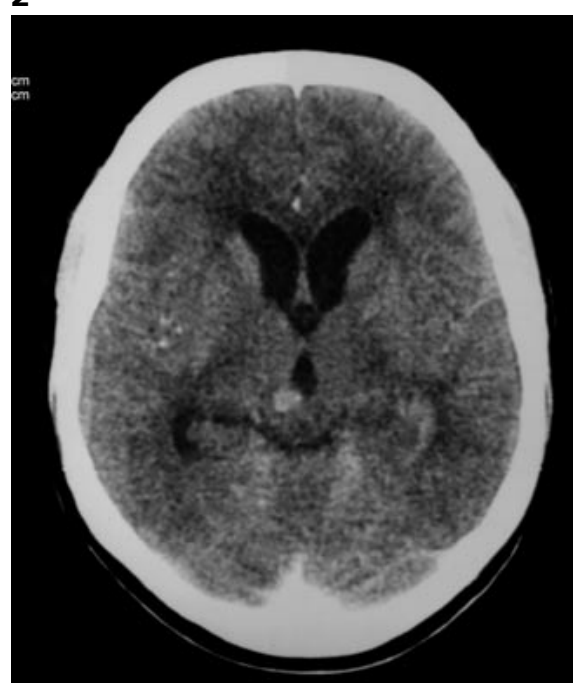

dence and computed tomographic (CT) patterns of proven cases of neurotuberculosis, both in Kuwaiti nationals and expatriates.

\section{Subjects and Methods}

A retrospective study of the case files and CT findings of 20 patients with proven ICTb was undertaken. These patients presented to the medical services of Al-Adan Hospital, a large general hospital in Kuwait, from 1985 to 1999 . At presentation all the patients underwent pre- and postintravenous contrast axial CT scans of the brain, with $10 \mathrm{~mm}$ thickness and $10 \mathrm{~mm}$ spacing. Presenting clinical features and relevant laboratory results, including cerebrospinal fluid (CSF) analysis and tissue biopsy reports, were obtained from the case files. Patients' chest radiographs were reviewed for evidence of Tb.

The CT films of the brain were assessed for the following: (1) number, size and site of the tuberculous lesions; (2) pre-contrast density appearances, compared to normal brain tissue, and the pattern of enhancement after intravenous administration of the contrast medium, and (3) the presence and severity of associated perifocal oedema, mass effect, obstructive hydrocephalus $(\mathrm{OHC})$ and basal meningeal enhancement. Ring enhancement was described as an unbroken ring of enhancement surrounding an unenhanced central part of the lesion, while disc enhancement was described as a homogeneous enhancement of the entire lesion. Perifocal oedema was assessed to be grade I if the diameter of the oedema was less than half the diameter of the lesion, and grade II if it was more than half. Mass effect was recorded as grade I if there was just effacement of the surrounding CSF spaces (ventricles, sulci or cisterns) and grade II if there was an associated shift of midline structures and/or OHC.

With a high probability diagnosis of intracranial tuberculoma, the patients were placed on at least three of four antituberculous medications: rifampicin, isoniazid, streptomycin, or pyrazinamide. Follow-up CT scans were done 2-12 months after initiation of therapy and the findings were compared with clinical response to antituberculous therapy (ATT).

\section{Results}

ICTb was confirmed in all the patients based on clinical features, laboratory data, CT findings and response to ATT. There were $15(75 \%)$ males and $5(25 \%)$ females. Ages ranged from 5 to 88 years (mean $=38.8$ years). Thirteen $(65 \%)$ patients were Kuwaiti nationals, $6(30 \%)$ from the Indian subcontinent and 1 from Afghanistan.

In this study, $13(65 \%)$ patients had evidence of extracranial tuberculosis. Ten (50\%) had evidence of pulmonary $\mathrm{Tb}$ on their chest radiographs and $7(35 \%)$ had histologically confirmed $\mathrm{Tb}$ lymphadenitis. One patient had gastrointestinal and pelvic $\mathrm{Tb}$, another had renal $\mathrm{Tb}$ and a 3 rd had tuberculous spondylitis. The latter 2 patients also had associated pulmonary $\mathrm{Tb}$.

At presentation, $13(65 \%)$ patients had single intracranial tuberculous lesions and 7 (35\%) had multiple lesions with a total number of 51 tuberculomas. One female patient alone had 14 small lesions $(0.5-1.2 \mathrm{~cm}$ in size) with only mild perifocal oedema but no associated mass effect or OHC (fig. 1). She fully recovered after a course of ATT with complete resolution of the intracranial lesions on a follow-up CT study. Another male patient had 11 lesions (1-3 cm in size) which caused significant mass effect and OHC. He had associated pulmonary and renal $\mathrm{Tb}$, and expired 3 days following admission to hospital. Overall, the sizes of ICTb ranged from 0.5 to $5 \mathrm{~cm}$ $($ mean $=2.8 \mathrm{~cm})$. They were all discrete lesions except in 1 patient who had 4 small lesions that appeared conglomerate. 
Fig. 3. a NECT scan showing two isodense lesions, one in the left frontoparietal region, the other in the left occipital lobe compressing the left lateral ventricle. $\mathrm{OHC}$ is noted. b CECT shows ring enhancement pattern of both lesions.
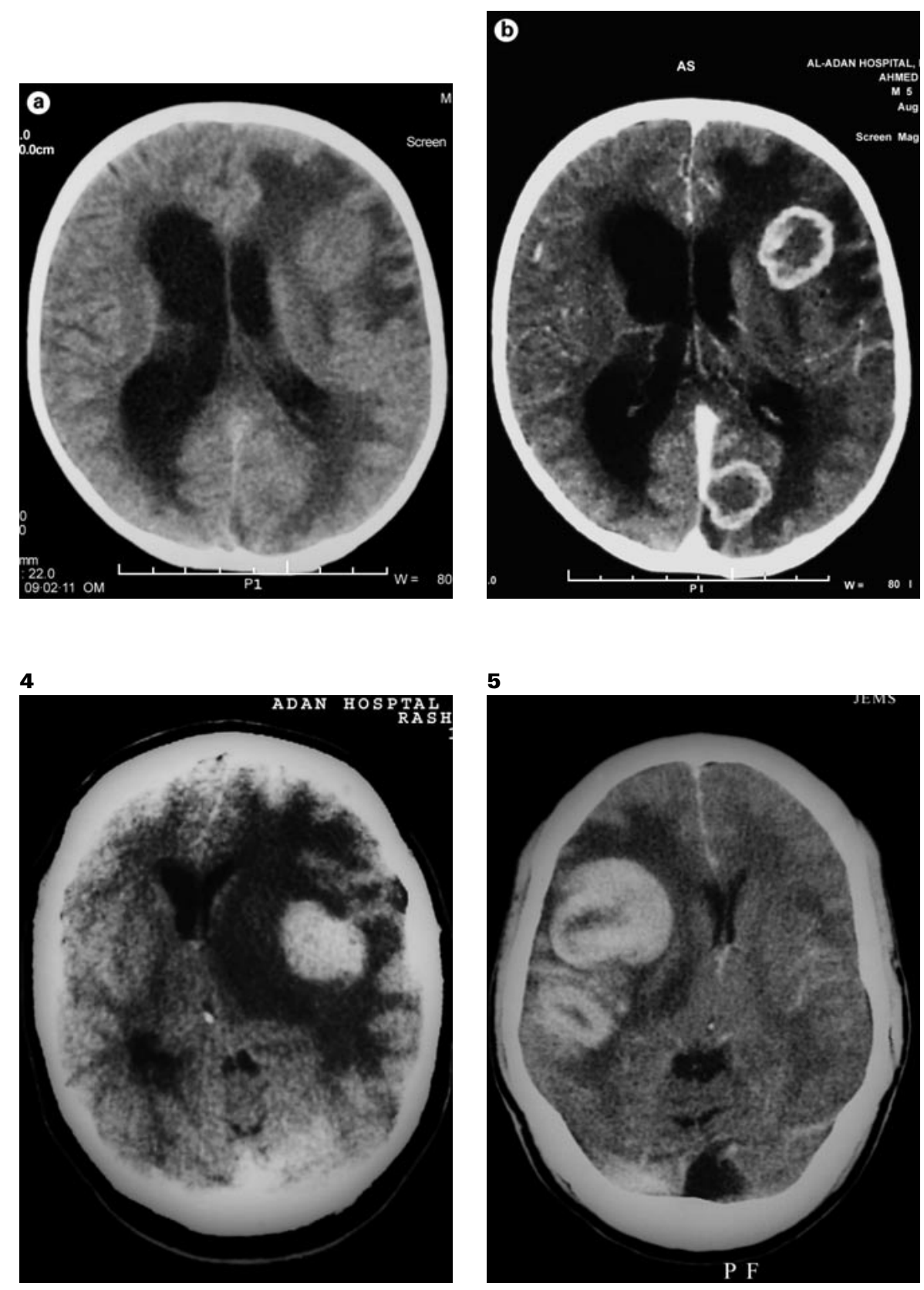

Fig. 4. CECT scan showing a disc-enhancing lesion in the left parietal lobe with marked perifocal oedema causing shift of the midline structures to the contralateral side.

Fig. 5. CECT shows two adjacent lesions in the right parietal lobe with the larger one showing combined disc and ring enhancement pattern.

In these patients, the majority (86\%) of the lesions were supratentorial, while only $14 \%$ were in the posterior fossa. Of the 51 tuberculomas, $40(78 \%)$ were located in the frontal and parietal lobes, $6(11.5 \%)$ in the cerebellum and 2 in the occipital lobe. Only one lesion was seen in the temporal region, one in the midbrain (fig. 2) and one in the basal ganglia (fig. 1).

In non-enhanced CT scans (NECT), 41 (81\%) lesions were isodense, 5 were hypodense and 4 were hyperdense.
In the contrast-enhanced CT scans (CECT), 27 (53\%) lesions showed ring enhancement (fig. 3), 21 (41\%) disc enhancement (fig. 4) and $3(6 \%)$ lesions combined ring and disc enhancement pattern on the initial CT scans (fig. 5). Of the 21 disc-enhanced lesions 14 were seen in 1 patient alone. Only one tuberculoma initially showed calcification and it appeared the same at follow-up CT studies. 
In general, the associated CT findings of grade II perifocal oedema and grade II mass effect were uncommon and were seen in only $3(15 \%)$ patients. However, grade I perifocal oedema was seen in $15(75 \%)$ patients and grade I mass effect in $9(45 \%)$. Meningeal enhancement was present in the CT scans of $7(35 \%)$ patients who also showed evidence of tuberculous meningitis in their CSF analysis, and 6 of them had OHC.

Clinical improvement on ATT alone was seen in 18 (90\%) of the 20 patients. The follow-up CT scans showed significant response to ATT in $16(80 \%)$ patients as evidenced by reduction in the number, size and surrounding oedema of the lesions. Only 1 patient, who was a 5-yearold mentally retarded child, died shortly after admission to hospital due to advanced multisystem disease. In another patient, the tuberculoma was removed surgically because the lesion was large with severe mass effect and OHC. Only in 2 patients did the appearance of the lesions remain almost the same despite clinical improvement with ATT. One of them had a calcified lesion and the other had residual hypodensity probably representing gliosis.

\section{Discussion}

Pulmonary $\mathrm{Tb}$ is the most common primary source of tubercle bacilli spreading to the brain $[1,3]$. In this study, it was the primary site in $50 \%$ of the patients, which is consistent with the $25-83 \%$ reported in the literature [2]. One of the patients was a known case of ICTb that was surgically excised but later presented with a new tuberculoma in another location within the brain, indicating reactivation from old quiescent lesions.

All expatriates undergo routine testing for pulmonary $\mathrm{Tb}$ in the form of chest X-ray as soon as they arrive in Kuwait. Only those who do not show evidence of active disease get the residence permit. However, the screening programme does not include neurotuberculosis. This might account for the presence of this disease in the expatriates. For Kuwaiti nationals, there are testing and vaccination programmes done routinely for all pre-school children. In spite of this, there has been an increased incidence of $\mathrm{Tb}$ in recent years, probably due to compromised immunity that might be related to drug addiction and HIV infection.

The frontal and parietal lobes were the major sites of preference of ICTb in these patients. Posterior fossa lesions, however, were relatively uncommon (14\%) but a little higher than the range of $6-10 \%$ reported in other studies [3, 6, 7]. Midbrain lesions are rare [7] and only one was observed in this study. Equally important is the fact that multiple tuberculomas have not been reported in the basal ganglia. In this study 1 patient had multiple tuberculomas located in the basal ganglia. Intraventricular lesions were not seen in our patients as Bhargava and Tandon [8] have pointed out.

The CT appearances of ICTb are non-specific and mimic a host of other diseases such as glioma, metastasis, neurocysticercosis, pyogenic abscess, infarction and haematoma $[9,10]$. The majority of the lesions $(81 \%)$ were isodense in NECT studies and many of these were visualized only after contrast medium administration. On the other hand, in CECT studies all lesions showed some form of enhancement. This means that non-contrast studies have low sensitivity for the detection of ICTb and that the administration of contrast medium is, therefore, mandatory for the evaluation of such patients. In this study, the tuberculous lesions showed one of three patterns of contrast enhancement. The ring and disc patterns of enhancement were described in previous reports $[4,8$, 10], while the combined ring and disc pattern seen in the initial CT scans of 3 of the patients has not been highlighted previously. The 'target sign' appearance described by other investigators [11-13] including those in Saudi Arabia [4], a country that shows similar demographic and ethnic patterns to Kuwait, was not seen in this study.

$\mathrm{OHC}$ results from involvement of the meninges by neurotuberculosis with the production of exudates or adhesions that block CSF passage through the basal cisterns and aqueduct of Sylvius [14]. The presence of $\mathrm{OHC}$ in our patients was significant enough to alter the approach to management.

ATT was started in most of the patients based on clinical suspicion, as previously reported $[14,15]$. The response to ATT was irrespective of the pattern of enhancement of the lesion or any other variable. In these patients there was no case of progressive enlargement of the lesion while on therapy, as was reported by Chambers et al. [12]. Paradoxical response to ATT has been reported on rare occasions early in the course of treatment $[16,17]$. These patients improve when corticosteroids are added to the regimen. The use of corticosteroids reduces the oedema around the tuberculoma decreasing the mass effect and thus giving ATT time to act [3].

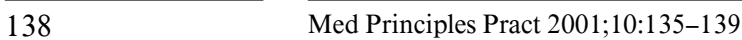




\section{Conclusion}

CT is a reliable modality in the diagnostic workup and follow-up of patients with ICTb. Not only does it help in evaluating patients' responses to ATT but it also helps to select patients who would require operative management. Administration of contrast medium is mandatory for the detection and proper assessment of the tuberculous lesions.

\section{References}

1 Asenjo A, Valladares H, Fierro J: Tuberculomas of the brain: Report of one hundred and fifty-nine cases. Arch Neurol Psychiatry 1951; 65:146-160

2 Dastur HM, Desai AD: A comparative study of brain tuberculoma and glioma based upon 107 case records of each. Brain 1965;88:375-396.

3 Abdul-Ghaffar NU, El-Sonbaty MR, Rahman NA: Intracranial tuberculoma in Kuwait. Int J Tuberc Lung Dis 1998;2:413-418.

4 Jinkins JR: Computed tomography of intracranial tuberculosis. Neuroradiology 1991;33: 126-135.

5 El-Sonbaty MR, Abdul-Ghaffar NA, Marafy AA: Multiple intracranial tuberculomas mimicking brain metastasis. Tuber Lung 1995;76: 271-272.

6 Selebler K, Erbengi A, Saribas O, Onol B: Giant calcified and ossified midbrain tuberculoma: Case report. J Neurosurg 1983;58:133135. 30:567-573.

\section{Acknowledgements}

The authors would like to thank Dr. Jasem Ramadan for his contribution in editing the manuscript, Mr. Girma Telahoun and Dr. G. Alex for their assistance in preparing the manuscript and Mrs. M. Vargese for typing it.
7 Mahanta A, Kalra L, Maheshwari ML, Mishra NK: Brain-stem tuberculoma: An unusual presentation. J Neurol 1982;227:249-253.

8 Bhargava S, Tandon PN: Intracranial tuberculomas: A CT study. Br J Radiol 1980;53:935945.

9 Hildebrandt G, Agnoli AL: Differential diagnosis and therapy of intracerebral tuberculomas. J Neurol 1982;228:201-208.

10 Welchman JM: Computerized tomography of intracranial tuberculomata. Clin Radiol 1979;

11 Van Dyk A: CT of intracranial tuberculomas with specific reference to the 'target sign'. Neuroradiology 1988;30:329-336.

12 Chambers ST, Hendrickse WA, Record C, Rudge P, Smith H: Paradoxical expansion of intracranial tuberculomas during chemotherapy. Lancet 1984;ii:181-183.
13 Traub M, Colchester AC, Kingsley DP, Swash $\mathrm{M}$ : Tuberculosis of the central nervous system. Q J Med (new series L III) 1984:53:81-100.

14 Dastur DK, Manghani DK, Udani PM: Pathology and pathogenetic mechanisms of neurotuberculosis. Radiol Clin North Am 1995;33: 733-752.

15 Tandon PN, Bhargava S: Effect of medical treatment on intracranial tuberculoma: A CT study. Tubercle 1985;66:85-97.

16 Abdul Jabbar M: Paradoxical response to chemotherapy for intracranial tuberculoma: Two case reports from Saudi Arabia. J Trop Med Hyg 1991;94:374-376.

17 Teoh R, Humphries MJ, O’Mahony G: Symptomatic intracranial tuberculoma developing during treatment of tuberculosis: A report of 10 patients and review of the literature. Q J Med 1987;63:449-460. 